IFP $/ 102 / \mathrm{UNC}$

\title{
GAUGE SYMMETRY IN BACKGROUND CHARGE CONFORMAL FIELD THEORY*
}

\author{
L. Dolan \\ Department of Physics and Astronomy,University of North Carolina \\ Chapel Hill, North Carolina 27599-3255, USA
}

\begin{abstract}
We present a mechanism to construct four-dimensional charged massless Ramond states using the discrete states of a fivebrane Liouville internal conformal field theory. This conformal field theory has background charge, and admits an inner product which allows positive norm states. A connection among supergravity soliton solutions, Liouville conformal field theory, non-critical string theory and their gauge symmetry properties is given. A generalized construction of the $S U(2)$ super Kac-Moody algebra mixing with the $N=1$ super Virasoro algebra is analyzed. How these Ramond states evade the DKV no-go theorem is explained.
\end{abstract}

October 1996

Submitted to Nuclear Physics B

* Supported in part by the U.S. Department of Energy under Grant No. DE-FG 05-85ER40219/Task A 


\section{Introduction}

The descriptions of string theory given by 1) solutions of low-energy classical target space supergravity theories, including non-perturbative BPS solitons, 2) worldsheet sigma model actions, and 3) conformal field theories of vertex operators have mutually reinforced our understanding of the spectrum and interactions of the system. In particular, duality relations among strong and weak coupling limits of various string models, and the presence of BPS non-perturbative states, some of which are massless, have signalled the importance of non-perturbative effects in our current formulation of string theory.

In this paper we analyze aspects of gauge symmetry in string theory using conformal field theories which already incorporate non-perturbative information, i.e. conformal field theories involving a coordinate field with background charge, such as the Liouville superfield. As a definite example, we consider the magnetically charged fivebrane soliton solution of the Type II string and its corresponding conformal field theory with background charge ${ }^{[1-5]}$. We demonstrate how the role of gauge symmetry differs in these theories from the action of gauge symmetry in critical string theory.

In particular, we show how the discrete states of the Liouville theory can be used to construct four-dimensional massless Ramond states which carry non-trivial charge under the non-abelian gauge symmetry of an $S U(2)$ super Kac-Moody algebra present on this Ramond side. We explain how this construction evades the arguments of the DKV no-go theorem.

Conventional superstring spectra given by conformal field theories can be changed by allowing non-vanishing vacuum expectation values (vevs) for any field in the low-energy supergravity, rather than only for the metric tensor. The vevs are given by classical solutions, either elementary or solitonic, of the supergravity field equations.

Soliton solutions are non-perturbative, and exist in both gauge theories and string theory. In particular, in Type II string models, they lead to states which enhance the original spectrum. This is interesting in that Type II was viewed as an economical model, but one which appeared to fall just short of phenomenological viability. Now with the inclusion of solitons, these theories are seen to have as many states as the heterotic string, by the mere choice of an appropriate internal space to compactify to four dimensions ${ }^{[6,7]}$. In this paper, we investigate an origin of spectrum enhancement present already in a conformal field theory (CFT) description.

The bosonic supergravity fields, apart from the metric, are described by d-form potentials, which fall into two categories: they are either NS-NS or RR, the latter occurring only in Type II superstrings. These two types are distinguished by the d-forms representing massless states having their origin in the Neveu-Schwarz/Neveu-Schwarz (NS) or the Ramond/Ramond (R) sector of the string theory.

Since RR-branes couple to RR field potentials, the RR-branes carry charge of the RR bosons. They have been dubbed Dirichlet p-branes, as they can be shown to correspond to states in the conformal field theory of type I string theory with Dirichlet boundary conditions ${ }^{[8,9]}$, where the open string ends live on D-branes. 
The fivebrane soliton we study is an NS-NS brane, and it describes a structure which carries charge under the NS-NS gauge bosons of the Type II superstring. In a limit corresponding to the semi-wormhole throat, the corresponding sigma model is known to be a superconformal field theory. Here we analyze its primary field content and the nature of its gauge symmetry currents. In sect.2, we briefly review the global $N=4$ conformal field theory description of the fivebrane soliton and discuss the primary fields of the $N=1$ subalgebra, which is local. In sect.3, the degrees of freedom of a $d=4$ string model, whose internal degrees of freedom can form global extended worldsheet symmetries, are given. The ghost system has conformal charge $c=-15$ on each side. In sect.4, the highest weight states and corresponding vertex operators of the $N=4$ superconformal algebra for both massive and massless (short) representations are computed. In sect.5, we give the fivebrane conformal field theory representation of the superconformal current algebra, which describes gauge symmetry in string theory. The non-zero charge of massless fermion representations is calculated. Eq.'s (5.9)-(5.17) contain the precise statement explaining how these states can exist. Conclusions and comments are found in sect.6. The appendix contains the operator product algebra of the $N=4$ superconformal theory.

\section{Conformal field theory with background charge}

The solitonic fivebrane solution ${ }^{[1-3]}$ in sigma model coordinates corresponding to the semi-wormhole throat has a metric given by

$$
d s^{2}=\eta_{M N} d x^{M} d x^{N}+\left(\frac{k_{6}}{y^{2}} e^{C_{0}}\right) \delta_{m n} d y^{m} d y^{n}
$$

where $0 \leq M, N \leq 5$, and $6 \leq m, n \leq 9$. The first term corresponds to a $c=9 \mathrm{CFT}$, and the second term represents a $c=6 \mathrm{CFT}$, where $k_{6}$ and $C_{0}$ are certain constants which contain a parameter $k$, the axion charge. We note that the (M,N) space-time part of the metric is flat, so that the $c=9$ part is a free conformal field theory ${ }^{[1-3]}$.

The $c=6$ part is a nontrivial conformal field theory, and we call it the $c=6$ "fivebrane" conformal field theory ${ }^{[4,5]}$. This conformal model is given by the following: It contains four affine Kac-Moody currents $J^{A}$ of dimension one satisfying an $U(1) \times S U(2)$ KMA (of level $k$ ) together with a set of four dimension- $\frac{1}{2}$ fields $\psi^{A}$ satisfying the free fermion algebra:

$$
\begin{aligned}
J^{0}(z) J^{0}(\zeta) & =-(z-\zeta)^{-2}+\ldots \\
J^{0}(z) J^{i}(\zeta) & =O(z-\zeta)^{0} \\
J^{i}(z) J^{j}(\zeta) & =-\frac{k}{2}(z-\zeta)^{-2}+\epsilon_{i j k} J^{k}(\zeta)(z-\zeta)^{-1}+\ldots \\
\psi^{A}(z) \psi^{B}(\zeta) & =-(z-\zeta)^{-1} \delta^{A B}+\ldots \\
\psi^{A}(z) J^{B}(\zeta) & =O(z-\zeta)^{0}
\end{aligned}
$$

The energy-momentum tensor from the Sugawara construction is:

$$
\tilde{L}(z)=-\frac{1}{2} J^{0} J^{0}-\frac{1}{k+2} J^{i} J^{i}-\frac{1}{2} \partial \psi^{A} \psi^{A}+\delta \frac{1}{4 z^{2}}
$$


where $\delta=0,1$ in the NS or R sector respectively; and $A=(0, i)$. The supercurrent is

$$
\tilde{F}(z)=\psi^{0} J^{0}+\frac{\sqrt{2}}{\sqrt{k+2}} \psi^{i} J^{i}+\frac{\sqrt{2}}{6 \sqrt{k+2}} \epsilon_{i j k} \psi^{i} \psi^{j} \psi^{k}
$$

The superconformal system $(2.3)$ has $\tilde{c}=\frac{6(k+1)}{(k+2)}$. In order to achieve the $c=6$ system corresponding to the fivebrane, a shifted CFT is defined from (2.3):

$$
\begin{aligned}
& \bar{L}(z)=\tilde{L}(z)+\frac{1}{2} Q \partial J^{0}(z) \\
& \bar{F}(z)=\tilde{F}(z)-Q \partial \psi^{0}(z)
\end{aligned}
$$

The superconformal system (2.3) has $c=\tilde{c}+3 Q^{2}$. We choose the background charge $Q^{2}=\frac{2}{k+2}$, so that $c=6$. Since we choose a real value for $Q$, this corresponds to the strong coupling region of Liouville theory ${ }^{[10,11]}$.

We can now construct the standard $N=4$ superconformal global algebra with generators $\bar{L}, \bar{F}^{A}, \overline{\mathcal{S}}^{i}$ where $\overline{\mathcal{S}}^{i}$ generate a global affine $S U(2)$ level $n$ algebra, and $c=6 n$. The semi-wormhole representation for this algebra has $c=6$, i.e. $n=1$ and is given by

$$
\begin{aligned}
& \bar{L}(z)=-\frac{1}{2} J^{0} J^{0}-\frac{1}{k+2} J^{i} J^{i}-\frac{1}{2} \partial \psi^{A} \psi^{A}+\delta \frac{1}{4 z^{2}}+\frac{1}{2} Q \partial J^{0} \\
& \bar{F}(z)=\psi^{0} J^{0}+\frac{\sqrt{2}}{\sqrt{k+2}} \psi^{i} J^{i}+\frac{\sqrt{2}}{6 \sqrt{k+2}} \epsilon_{i j k} \psi^{i} \psi^{j} \psi^{k}-Q \partial \psi^{0} \\
& \bar{F}^{i}(z)=\psi^{i} J^{0}+\frac{\sqrt{2}}{\sqrt{k+2}}\left[-\psi^{0} J^{i}+\epsilon_{i j k} J^{j} \psi^{k}-\frac{1}{2} \epsilon_{i j k} \psi^{0} \psi^{j} \psi^{k}\right]-Q \partial \psi^{i} \\
& \overline{\mathcal{S}}^{i}(z)=\frac{1}{2}\left(\psi^{0} \psi^{i}+\frac{1}{2} \epsilon_{i j \ell} \psi^{j} \psi^{\ell}\right)
\end{aligned}
$$

for $\delta=0,1$ for NS or R sector respectively. We set $Q=-\frac{\sqrt{2}}{\sqrt{k+2}}$ and the currents in (2.5) close on the $N=4$ superconformal algebra given in appendix A. For $\bar{L}(z)$ hermitian, i.e. $\bar{L}_{n}^{\dagger}=\bar{L}_{-n}$, then $J_{n}^{0 \dagger}=-J_{n}^{0}$ for $n \neq 0, J_{0}^{0 \dagger}=-Q-J_{0}^{0}, \psi_{n}^{A \dagger}=-\psi_{-n}^{A}$, and $J_{n}^{i \dagger}=-J_{-n}^{i}$. Therefore the states $J_{-n}^{0}|\psi\rangle$ for $n>0$ have positive norm:

$$
\left.\| J_{-n}^{0}|\psi\rangle\left\|^{2}=\left\langle\psi\left|\left(-J_{n}^{0}\right) J_{-n}^{0}\right| \psi\right\rangle=n\langle\psi \mid \psi\rangle=n\right\| \| \psi\right\rangle \|^{2}>0
$$

for $\||\psi\rangle \|^{2}>0$. A similar argument holds for $\psi_{-n}^{A}|\psi\rangle$ and $J_{-n}^{i}|\psi\rangle$. It follows from the above hermiticity conditions on $J_{n}^{A}, \psi_{n}^{A}$ that $\bar{F}_{n}^{\dagger}=\bar{F}_{-n}, \bar{F}_{n}^{i \dagger}=\bar{F}_{-n}^{i}$, and $\overline{\mathcal{S}}_{n}^{i \dagger}=-\overline{\mathcal{S}}_{-n}^{i}$.

In the string models we consider, with the conformal field theory in (2.2) as a building block, we will use an $N=1$ superconformal ghost system, so the $N=4$ algebra serves as a global worldsheet algebra, which has highest weight representations ${ }^{[12]}$. Its $N=1$ subalgebra $\bar{L}, \bar{F}$ will be used as the matter (i.e. non-ghost) contribution to the BRST charge operator. We note that although $\overline{\mathcal{S}}^{i}(z)$ in $(2.5)$ is a primary conformal field, it is not the upper component of a primary superfield under the $N=1$ subalgebra. In 
order to study vertex operators, we consider the primary weight one-half superfield whose components are

$$
V_{L}^{i}(z)=\psi^{i} ; \quad V_{U}^{i}(z)=Q\left(\frac{1}{2} \epsilon_{i j k} \psi^{j} \psi^{k}+J^{i}\right) \equiv T^{i}
$$

where

$$
\begin{aligned}
& \bar{F}(z) \psi^{i}(\zeta)=(z-\zeta)^{-1} T^{i}(\zeta) \\
& \bar{F}(z) T^{i}(\zeta)=(z-\zeta)^{-2} \psi^{i}(\zeta)+(z-\zeta)^{-1} \partial \psi^{i}(\zeta)
\end{aligned}
$$

We define $T^{i} \equiv V_{U}^{i}$ and (2.8) forms a representation of a super Kac-Moody algebra

$$
\begin{aligned}
T^{i}(z) T^{j}(\zeta) & =-\frac{\delta_{i j}}{(z-\zeta)^{2}}+\frac{Q \epsilon_{i j k} T^{k}(\zeta)}{(z-\zeta)} \\
T^{i}(z) \psi^{j}(\zeta) & =\frac{Q \epsilon_{i j k} \psi^{k}(\zeta)}{(z-\zeta)} \\
\psi^{i}(z) \psi^{j}(\zeta) & =-\frac{\delta_{i j}}{(z-\zeta)} .
\end{aligned}
$$

The level of the KMA $T^{i}$ is $k+2$. If we bosonize the $J^{0}$ current by $J^{0}(z)=-\partial \phi(z)$, where $\phi(z) \phi(\zeta)=-\ln (z-\zeta)+\ldots$ for $|z|>|\zeta|$, then the conformal field : $e^{\beta \phi(z)}$ : with $: e^{\beta \phi(0)}:|0\rangle=|\beta\rangle$ is primary with respect to $\bar{L}(z)$ with conformal weight $h_{Q}=-\frac{1}{2} \beta(\beta+Q)$. The operator product

$$
J^{0}(z): e^{\beta \phi(\zeta)}:=\beta: e^{\beta \phi(\zeta)}:(z-\zeta)^{-1}+\ldots
$$

implies $J_{0}^{0}|\beta\rangle=\beta|\beta\rangle$. The anomalous product

$$
\bar{L}(z) J^{0}(\zeta)=Q(z-\zeta)^{-3}+J^{0}(\zeta)(z-\zeta)^{-2}+\partial J^{0}(\zeta)(z-\zeta)^{-1}+\ldots
$$

implies $\left[L_{n}, J_{m}\right]=-m J_{n+m}+\frac{Q}{2} n(n+1) \delta_{n,-m}$. For a CFT with background charge, considering the moments of the current $J^{0}(z)$ and requiring $\bar{L}_{n}^{\dagger}=\bar{L}_{-n}$, we derive

$$
J_{0}^{0 \dagger}=-\left[\bar{L}_{-1}, J_{1}^{0}\right]^{\dagger}=-\left[\bar{L}_{1}, J_{-1}^{0}\right]=-J_{0}^{0}-Q
$$

The allowed values for $\beta$ such that $h_{Q}$ is real are given below (4.11). The case we consider in this paper is real $Q$ and real $h_{Q}$.

We now discuss in detail an inner product for the Liouville states with real $\beta$, i.e. the discrete states, by describing an inner product for the vector space of states whose elements, i.e. vectors, are linear combinations of the states $|\beta\rangle$, including the unique element $\mathbf{0}$ defined by $|\beta\rangle-|\beta\rangle=\mathbf{0}$. We note $|0\rangle$ is different from $\mathbf{0}$. The choice of an inner product in a vector space is by no means uniquely determined by the vector space. The choice of an inner product is a free choice, which leads us to consider the question of exactly what is chosen, i.e. what is an inner product. ${ }^{[13]}$ 
An inner product on a real vector space is a real-valued function which assigns to any ordered pair of vectors such as $|\beta\rangle,\left|\beta^{\prime}\right\rangle$ a real number, denoted by $\left(\left|\beta^{\prime}\right\rangle,|\beta\rangle\right)$ and satisfying i) $\left(\left|\beta^{\prime}\right\rangle,|\beta\rangle\right)=\left(|\beta\rangle,\left|\beta^{\prime}\right\rangle\right)$, i.e. the inner product is symmetric, ii) $\left(\left|\beta^{\prime}\right\rangle, a|\beta\rangle\right)=a\left(\left|\beta^{\prime}\right\rangle,|\beta\rangle\right)$, for any scalar $a$, iii) $\left(\left|\beta^{\prime}\right\rangle,\left[\left|\beta_{1}\right\rangle+\left|\beta_{2}\right\rangle\right]\right)=\left(\left|\beta^{\prime}\right\rangle,\left|\beta_{1}\right\rangle\right)+\left(\left|\beta^{\prime}\right\rangle,\left|\beta_{2}\right\rangle\right)$, iv $)(|\beta\rangle,|\beta\rangle)>0$, and v) $(\mathbf{0}, \mathbf{0})=0,(\mathbf{0},|\beta\rangle)=(|\beta\rangle, \mathbf{0}),(\mathbf{0}, a|\beta\rangle)=a(\mathbf{0},|\beta\rangle)$, for any scalar $a$, and $\left(\mathbf{0},\left[\left|\beta_{1}\right\rangle+\left|\beta_{2}\right\rangle\right]\right)=$ $\left(\mathbf{0},\left|\beta_{1}\right\rangle\right)+\left(\mathbf{0},\left|\beta_{2}\right\rangle\right)$.

We now choose a particular inner product for the Liouville states $|\beta\rangle$. We define the inner product $\left(\left|\beta^{\prime}\right\rangle,|\beta\rangle\right)$ such that

$$
\left(\left|\beta^{\prime}\right\rangle, J_{0}^{0}|\beta\rangle\right)=\left(J_{0}^{0 \dagger}\left|\beta^{\prime}\right\rangle,|\beta\rangle\right)
$$

where the lefthand side of (2.12) is

$$
\left(\left|\beta^{\prime}\right\rangle, J_{0}^{0}|\beta\rangle\right)=\beta\left(\left|\beta^{\prime}\right\rangle,|\beta\rangle\right) .
$$

The righthand side of $(2.12)$ is

$$
\left(J_{0}^{0 \dagger}\left|\beta^{\prime}\right\rangle,|\beta\rangle\right)=\left(\beta^{\prime}\left|\beta^{\prime}\right\rangle,|\beta\rangle\right) .
$$

The choice of inner product is defined by (2.14). It is equivalent to defining the adjoint of the state $|\beta\rangle$ to be $\langle-\beta-Q|$ in the sense that

$$
\begin{aligned}
J_{0}^{0}|\beta\rangle & =\beta|\beta\rangle \\
{\left[J_{0}^{0}|\beta\rangle\right]^{\dagger} } & =\beta\langle-\beta-Q| .
\end{aligned}
$$

So that in $(2.12)$ the righthand side $\left(J_{0}^{0 \dagger}\left|\beta^{\prime}\right\rangle,|\beta\rangle\right)$ means $\left(\left[\left\langle-\beta^{\prime}-Q\right| J_{0}^{0}\right]^{\dagger},|\beta\rangle\right)$. Here (2.15b) for $\beta=\beta^{\prime}$, which is $\left\langle-\beta^{\prime}-Q\right| J_{0}^{0 \dagger}=\beta^{\prime}\left\langle-\beta^{\prime}-Q\right|$, is the definition used in deriving (2.14), i.e. our choice for inner product is

$$
\left(\left|\beta^{\prime}\right\rangle,|\beta\rangle\right) \equiv\left\langle-\beta^{\prime}-Q \mid \beta\right\rangle
$$

This choice satisfies the conditions $\mathrm{i})-\mathrm{v}$ ) listed above. From (2.12)-(2.14), we see that $\left(\left|\beta^{\prime}\right\rangle,|\beta\rangle\right)$ is non-vanishing only for $\beta=\beta^{\prime}$, and we normalize $(|\beta\rangle,|\beta\rangle)=1$. In the bra, ket notation above, this is

$$
\langle-\beta-Q \mid \beta\rangle=1
$$

We also have

$$
\left\langle 0\left|e^{-Q \phi(z)}\right| 0\right\rangle=1 .
$$

With the inner product defined by (2.14), the discrete Liouville states have positive norm:

$$
\| \beta\rangle \|^{2} \equiv(|\beta\rangle,|\beta\rangle)=1
$$

In sect. 5, we consider the holomorphic $N=1 S U(2)$ super Kac-Moody algebra mixing with this CFT and show how these discrete states allow for massless Ramond states (4d 
spacetime fermions) which carry non zero $S U(2)$ charge on a given side of a closed string theory.

\section{The string model}

We consider compactification of the Type II superstring with four-dimensional Lorentz invariance. There is a $c=9, N=1$ internal (local) superconformal algebra (SCA) associated with any such compactification. For theories with $d=4, N=2$ spacetime supersymmetry, the spacetime supersymmetry implies that this internal SCA splits into a $c=6$ piece with $N=4$ superconformal (global) symmetry and a $c=3$ piece with $N=2$ superconformal (global) symmetry. ${ }^{[14,15]}$ Here we use local to mean the BRST ghost system also satifies this algebra.

We consider the $M^{(4)} \otimes T^{(2)} \otimes W_{k}^{(4)}$ model, where $W_{k}^{(4)}$ is the $c=6$ fivebrane CFT given in (2.4), and $M^{(4)} \otimes T^{(2)}$ is the flat four-dimensional non-compact flat Minkowski spacetime times a two-dimensional compact flat torus, given in terms of six free superfields $M=(0 \leq \mu \leq 3 ; 4 \leq m \leq 5)$,

$$
\begin{aligned}
a^{M}(z) a^{N}(\zeta) & =\eta^{M N}(z-\zeta)^{-2}+\ldots \\
\psi^{M}(z) \psi^{N}(\zeta) & =\eta^{M N}(z-\zeta)^{-1}+\ldots \\
\psi^{M}(z) a^{N}(\zeta) & =0
\end{aligned}
$$

where one of the free superfields is chosen to be timelike, and the others to be Euclidean (spacelike); $\eta^{M N}=(-1,1,1,1,1,1)$ and $a^{M}(z) \equiv i \partial X^{M}(z)$ where

$$
\begin{aligned}
X^{M}(z) X^{N}(\zeta) & =-\eta^{M N} \ln (z-\zeta)+: X^{M}(z) X^{N}(\zeta): \\
\psi^{M}(z) \psi^{N}(\zeta) & =\eta^{M N}(z-\zeta)^{-1}+\ldots \\
\psi^{M}(z) a^{N}(\zeta) & =O(z-\zeta)^{0}
\end{aligned}
$$

Then

$$
\begin{aligned}
& L(z)=\frac{1}{2}: a^{M} a_{M}:+\frac{1}{2} \partial \psi^{M} \psi_{M}-\frac{1}{2} J^{0} J^{0}-\frac{1}{2} Q^{2} J^{i} J^{i}-\frac{1}{2} \partial \psi^{A} \psi^{A}+\delta \frac{5}{8 z^{2}}+\frac{1}{2} Q \partial J^{0} \\
& F(z)=a^{M} \psi_{M}+\psi^{0} J^{0}-Q \psi^{i} J^{i}-\frac{Q}{6} \epsilon_{i j k} \psi^{i} \psi^{j} \psi^{k}-Q \partial \psi^{0}
\end{aligned}
$$

for $\delta=0,1$ for NS or R sector respectively, where $Q=-\frac{\sqrt{2}}{\sqrt{k+2}}$ and $c=15$.

\section{Unitary representations of the $N=4$ superconformal algebra}

\section{NEVEU-SCHWARZ STATES}

We analyze the BRST properties ${ }^{[16]}$ and gauge symmetry of the following fourdimensional vertex operators, given here in covariant form where $\mu$ labels spacetime, 
$0 \leq \mu \leq 3$, and the metric is $\eta_{\mu \nu}=\operatorname{diag}(-1,1,1,1)$. The weight one primary conformal fields for Neveu-Schwarz states with one oscillator are given in the superconformal ghost number $q=-1$ picture by

$$
\begin{aligned}
V_{-1}(k, z, \epsilon) & =\epsilon \cdot \psi(z) e^{i k_{\mu} X^{\mu}(z)} e^{\beta \phi(z)} V_{j m}(z) e^{-\varphi_{\mathrm{gh}}(z)} \\
V_{-1}^{i}(k, z) & =\psi^{i}(z) e^{i k_{\mu} X^{\mu}(z)} e^{\beta \phi(z)} V_{j m}(z) e^{-\varphi_{\mathrm{gh}}(z)} \\
V_{-1}^{0}(k, z) & =\psi^{0}(z) e^{i k_{\mu} X^{\mu}(z)} e^{\beta \phi(z)} V_{j m}(z) e^{-\varphi_{\mathrm{gh}}(z)} \\
V_{-1}^{n}(k, z) & =\psi^{n}(z) e^{i k_{\mu} X^{\mu}(z)} e^{\beta \phi(z)} V_{j m}(z) e^{-\varphi_{\mathrm{gh}}(z)},
\end{aligned}
$$

where the weight one condition implies $k_{\mu} k^{\mu}-\beta(\beta+Q)+Q^{2} j(j+1)=0$ for each field. Also $1 \leq n \leq 6$ where the $\psi^{n}$ form a $c=3$ superconformal system corresponding to two supercoordinates $\psi^{\ell}+\theta a^{\ell}, \ell=1,2$ compactified on $T^{2}$. The fields $V_{j m}(z)$, which are primary under the superconformal algebra as well as the level $k \mathrm{SU}(2)$ affine algebra, form $S U(2)$ multiplets with $m=(-j,-j+1, \ldots, j)$. Here $V_{j m}(z)$ corresponds to a unitary highest weight representation of affine $S U(2)_{k}$, so that $j=0, \frac{1}{2}, 1, \ldots, \frac{k}{2}$.

$$
\begin{aligned}
L(z) V_{j m}(\zeta) & =(z-\zeta)^{-2} \frac{j(j+1)}{k+2} V_{j m}(\zeta)+(z-\zeta)^{-1} \partial_{\zeta} V_{j, m}(\zeta) \\
J^{i}(z) V_{j m}(\zeta) & =(z-\zeta)^{-1} f_{i m m^{\prime}} V_{j m}(\zeta)
\end{aligned}
$$

where $f_{i m m^{\prime}}=t_{m m^{\prime}}^{i}$ is the $S U(2)$ representation of $V_{j m}(\zeta)$. Without the ghosts, states in the Neveu-Schwarz system form superconformal fields $V(z, \theta)=V_{q}(z)+\theta V_{q+1}(z)$ with upper and lower components related by

$$
\begin{aligned}
G(z) V_{q}(\zeta) & =(z-\zeta)^{-1} V_{q+1}(\zeta) \\
G(z) V_{q+1}(\zeta) & =(z-\zeta)^{-2} 2 h_{q} V_{q}(\zeta)+(z-\zeta)^{-1} \partial V_{q}(\zeta) .
\end{aligned}
$$

The BRST current for the $c=15$ superVirasoro currents $L, F$ is the usual expression

$$
\begin{aligned}
Q(z) & =Q_{0}(z)+Q_{1}(z)+Q_{2}(z) \\
Q_{0}(z) & =c L-{ }_{\times}^{\times} c b \partial c_{\times}^{\times}+\frac{3}{2} \partial^{2} c+c L^{\beta \gamma}+\partial\left(\frac{3}{4} \times{ }_{\times} \gamma \beta_{\times}^{\times}\right) \\
Q_{1}(z) & =-\frac{1}{2} e^{\left(\chi_{\mathrm{gh}}+\varphi_{\mathrm{gh}}\right)} F \\
Q_{2}(z) & =-\frac{1}{4} \gamma b \gamma .
\end{aligned}
$$

BRST invariance holds for the vertices (4.1) with the following restrictions. For

$$
F(z)=a_{\mu}(z) \psi^{\mu}(z)+\bar{F}(z)+a_{\ell}(z) \psi^{\ell}(z)
$$

and

$$
\bar{F}(z)=\psi^{0} J^{0}-Q \psi^{i} J^{i}-Q \frac{1}{6} \epsilon_{i j k} \psi^{i} \psi^{j} \psi^{k}-Q \partial \psi^{0}
$$


where the background charge is $Q=-\sqrt{\frac{2}{2+k}}$, we have

$$
\begin{aligned}
& F(z) V_{-1}(k, \zeta, \epsilon)=(z-\zeta)^{-1}\left[\left(k \cdot \psi(\zeta) \epsilon \cdot \psi(\zeta)+\epsilon \cdot a(\zeta)+\beta \psi^{0}(\zeta) \epsilon \cdot \psi(\zeta)\right) V_{j m}(\zeta)\right. \\
&\left.-Q \psi^{i}(\zeta) \epsilon \cdot \psi(\zeta) f_{i m m^{\prime}} V_{j m^{\prime}}(\zeta)\right] e^{i k_{\mu} X^{\mu}(\zeta)} e^{\beta \phi(\zeta)} \\
& F(z) V_{-1}^{i}(k, \zeta)=(z-\zeta)^{-2}\left[Q f_{i m m^{\prime}} V_{j m^{\prime}}(\zeta)\right] e^{i k_{\mu} X^{\mu}(\zeta)} e^{\beta \phi(\zeta)} \\
&+(z-\zeta)^{-1}\left[\left(k \cdot \psi(\zeta) \psi^{i}(\zeta)+\beta \psi^{0}(\zeta) \psi^{i}(\zeta)+Q \frac{1}{2} \epsilon_{i k k^{\prime}} \psi^{k}(\zeta) \psi^{k^{\prime}}(\zeta)\right) V_{j m}(\zeta)\right. \\
&\left.+Q\left(: J^{i}(\zeta) V_{j m}(\zeta):-\psi^{k}(\zeta) \psi^{i}(\zeta) f_{k m m^{\prime}} V_{j m^{\prime}}(\zeta)\right)\right] e^{i k_{\mu} X^{\mu}(\zeta)} e^{\beta \phi(\zeta)} \\
& F(z) V_{-1}^{0}(k, \zeta)=(z-\zeta)^{-2}\left[(-\beta-Q) V_{j m}(\zeta)\right] e^{i k_{\mu} X^{\mu}(\zeta)} e^{\beta \phi(\zeta)} \\
&+(z-\zeta)^{-1}\left[\left(k \cdot \psi(\zeta) \psi^{0}(\zeta) e^{\beta \phi(\zeta)}+: \partial \phi(\zeta) e^{\beta \phi(\zeta)}:\right) V_{j m}(\zeta)\right. \\
&\left.-Q \psi^{k}(\zeta) \psi^{0}(\zeta) f_{k m m^{\prime}} V_{j m^{\prime}}(\zeta) e^{\beta \phi(\zeta)}\right] e^{i k_{\mu} X^{\mu}(\zeta)} \\
& F(z) V_{-1}^{n}(k, \zeta)=(z-\zeta)^{-1}\left[\left(k \cdot \psi(\zeta) \psi^{n}(\zeta)+\beta \psi^{0}(\zeta) \psi^{n}(\zeta)-\frac{1}{2} \epsilon_{n n^{\prime} n^{\prime \prime}} \psi^{n^{\prime}}(\zeta) \psi^{n^{\prime \prime}}(\zeta)\right) V_{j m}(\zeta)\right. \\
&\left.-Q \psi^{k}(\zeta) \psi^{n}(\zeta) f_{k m m^{\prime}} V_{j m^{\prime}}(\zeta)\right] e^{i k_{\mu} X^{\mu}(\zeta)} e^{\beta \phi(\zeta)} .
\end{aligned}
$$

In (4.7), the four expressions $V_{-1}$ are used without the ghost field. BRST invariance of the vertex operators is $\frac{1}{2 \pi i} \oint_{\zeta} d z Q(z) V_{-1}(\zeta)=0$ which thus requires $V_{j m}(z)=V_{00}(z)$ in $(4.1 \mathrm{~b})$ and $\beta=-Q$ in (4.1c). With these restrictions, we have $F_{s} V_{-1}(k, 0)|0\rangle=0$ for $s \geq \frac{1}{2}$; so that $V_{-1}(k, 0)|0\rangle$ satisfy the physical state conditions. We note that $\left[L_{-1}, V_{j m}(\zeta)\right]=$ $-Q^{2} f_{i m m^{\prime}}: J^{i}(\zeta) V_{j m}(\zeta):=\partial V_{j m}(\zeta)$. Therefore $\partial V_{00}=0$, so $V_{00}(\zeta)$ is a constant, and in what follows we can set it equal to one. We then define the upper components of (4.1), which satisfy (4.3) as

$$
\begin{aligned}
V_{0}(k, \zeta, \epsilon)= & {\left[\left(k \cdot \psi(\zeta) \epsilon \cdot \psi(\zeta)+\epsilon \cdot a(\zeta)+\beta \psi^{0}(\zeta) \epsilon \cdot \psi(\zeta)\right) V_{j m}(\zeta)\right.} \\
& \left.-Q \psi^{i}(\zeta) \epsilon \cdot \psi(\zeta) f_{i m m^{\prime}} V_{j m^{\prime}}(\zeta)\right] e^{i k_{\mu} X^{\mu}(\zeta)} e^{\beta \phi(\zeta)} \\
V_{0}^{i}(k, \zeta)= & {\left[k \cdot \psi(\zeta) \psi^{i}(\zeta)+\beta \psi^{0}(\zeta) \psi^{i}(\zeta)\right.} \\
& \left.+Q\left(J^{i}(\zeta)+\frac{1}{2} \epsilon_{i k k^{\prime}} \psi^{k}(\zeta) \psi^{k^{\prime}}(\zeta)\right)\right] e^{i k_{\mu} X^{\mu}(\zeta)} e^{\beta \phi(\zeta)} \\
V_{0}^{0}(k, z)= & {\left[\left(k \cdot \psi(\zeta) \psi^{0}(\zeta) e^{-Q \phi(\zeta)}+: \partial \phi(\zeta) e^{-Q \phi(\zeta)}:\right) V_{j m}(\zeta)\right.} \\
& \left.-Q \psi^{k}(\zeta) \psi^{0}(\zeta) f_{k m m^{\prime}} V_{j m^{\prime}}(\zeta) e^{-Q \phi(\zeta)}\right] e^{i k_{\mu} X^{\mu}(\zeta)} \\
V_{0}^{n}(k, z)= & {\left[\left(k \cdot \psi(\zeta) \psi^{n}(\zeta)+\beta \psi^{0}(\zeta) \psi^{n}(\zeta)-\frac{1}{2} \epsilon_{n n^{\prime} n^{\prime \prime}} \psi^{n^{\prime}}(\zeta) \psi^{n^{\prime \prime}}(\zeta)\right) V_{j m}(\zeta)\right.} \\
& \left.-Q \psi^{k}(\zeta) \psi^{n}(\zeta) f_{k m m^{\prime}} V_{j m^{\prime}}(\zeta)\right] e^{i k_{\mu} X^{\mu}(\zeta)} e^{\beta \phi(\zeta)}
\end{aligned}
$$

From (4.1) we see that the states $V_{-1}(k, 0)|0\rangle$ are tensor products of states in the three conformal field theories: ( spacetime cft $c=6) \otimes($ fivebrane $\operatorname{cft} c=6) \otimes\left(T^{2} \mathrm{cft} c=3\right.$ ). In order to specify the quantum numbers $\beta$ and $j$ in (4.1), we consider the conditions for highest weight representations ${ }^{[12]}$ of the global $N=4$ superconformal algebra constructed from the $c=6$ fivebrane CFT as given in (2.5) and Appendix A. In the Neveu-Schwarz sector, the highest weight states of the representations are annihilated by $\bar{L}_{n}, \bar{F}_{r}, \bar{F}_{r}^{i}, \overline{\mathcal{S}}_{n}^{i}, \overline{\mathcal{S}}_{0}^{+}$ for $n \geq 1, r \geq \frac{1}{2}$ and are labelled by

$$
\bar{L}_{0}|h, \ell\rangle=h|h, \ell\rangle, \quad i \overline{\mathcal{S}}_{0}^{3}|h, \ell\rangle=\ell|h, \ell\rangle,
$$


where $\ell=0$ and $h>0$ for massive representations; and $h=\ell$ with $\ell=0, \frac{1}{2}$ for massless representations ${ }^{[12]}$. For massless representations, the highest weight states $|h, \ell\rangle$ are also annihilated by $\overline{\mathcal{G}}_{-\frac{1}{2}}^{1}$ and $\mathcal{G}_{-\frac{1}{2}}^{2}$ with $\mathcal{G}^{a}$ given in Appendix A:

$$
\overline{\mathcal{G}}_{-\frac{1}{2}}^{1}|h, \ell\rangle=0 ; \quad \mathcal{G}_{-\frac{1}{2}}^{2}|h, \ell\rangle=0 .
$$

For states with no $(N=4)$ oscillators as in $(4.1 \mathrm{a}, \mathrm{d})$, we have

$$
\bar{L}_{0} e^{\beta \phi(0)} V_{j m}(0)|0\rangle=\left[-\frac{1}{2} \beta(\beta+Q)+\frac{1}{2} Q^{2} j(j+1)\right] e^{\beta \phi(0)} V_{j m}(0)|0\rangle .
$$

For $h_{Q} \equiv-\frac{1}{2} \beta(\beta+Q)$ to be real, either $\beta=-\frac{1}{2} Q+i y$ with real $y$ so that $h_{Q}=\frac{1}{8} Q^{2}+\frac{1}{2} y^{2}$ is positive; or $\beta$ is real. If $\beta$ is real, the highest weight conditions for massless representations require it to take on discrete values, a feature similar to the discrete states in $c=1$ matter coupled to $2 d$ quantum gravity, i.e. the Liouville field. ${ }^{[17,11]}$ Also

$$
i \overline{\mathcal{S}}_{0}^{3} e^{\beta \phi(0)} V_{j m}(0)|0\rangle=\frac{i}{2} \sum_{s}\left(\psi_{-s}^{0} \psi_{s}^{i}+\frac{1}{2} \epsilon_{i j \ell} \psi_{-s}^{j} \psi_{s}^{\ell}\right) e^{\beta \phi(0)} V_{j m}(0)|0\rangle=0
$$

so that $e^{\left(-\frac{Q}{2}+i y\right) \phi(0)} V_{j m}(0)|0\rangle$ correspond to $N=4$ highest weight massive states: $h>0$, $\ell=0$. The states $e^{\beta \phi(0)} V_{j m}(0)|0\rangle$ for $\beta=Q j,-Q(j+1)$ have $h=\ell=0$, and from (4.10) correspond to $N=4$ highest weight massless states for $\beta=Q j=0$.

For states with one $(N=4)$ oscillator as in $(4.1 \mathrm{~b}, \mathrm{c})$, we have

$$
\bar{L}_{0} \psi^{(i, 0) i}(0) e^{\beta \phi(0)} V_{j m}(0)|0\rangle=\left[\frac{1}{2}-\frac{1}{2} \beta(\beta+Q)+\frac{1}{2} Q^{2} j(j+1)\right] \psi^{(i, 0)}(0) e^{\beta \phi(0)} V_{j m}(0)|0\rangle .
$$

Also

$$
\begin{aligned}
& i \overline{\mathcal{S}}_{0}^{3}\left(\psi^{1}(0) \pm i \psi^{2}(0)\right) e^{\beta \phi(0)} V_{j m}(0)|0\rangle= \pm \frac{1}{2}\left(\psi^{1}(0) \pm i \psi^{2}(0)\right) e^{\beta \phi(0)} V_{j m}(0)|0\rangle \\
& i \overline{\mathcal{S}}_{0}^{3}\left(\psi^{3}(0) \mp i \psi^{0}(0)\right) e^{\beta \phi(0)} V_{j m}(0)|0\rangle= \pm \frac{1}{2}\left(\psi^{3}(0) \mp i \psi^{0}(0)\right) e^{\beta \phi(0)} V_{j m}(0)|0\rangle
\end{aligned}
$$

and we find that

$$
\left(\psi^{1}(0)+i \psi^{2}(0)\right) e^{-Q \phi(0)}|0\rangle ; \quad\left(\psi^{3}(0)-i \psi^{0}(0)\right) e^{-Q \phi(0)}|0\rangle
$$

satisfy the $N=4$ highest weight massless state conditions with $h=\ell=\frac{1}{2}$. The states $\psi^{i}(0) e^{\left(-\frac{Q}{2}+i y\right) \phi(0)}|0\rangle$ and $\psi^{0}(0) e^{\left(-\frac{Q}{2}+i y\right) \phi(0)}|0\rangle$ are not $N=4$ highest weight massive states since both $F_{s}, F_{s}^{i}$ acting on either of them is not zero, for all $s \geq \frac{1}{2}$. BRST invariant vertex operators comprised of worldsheet $N=4$ massive representations are

$$
\begin{aligned}
V_{-1}(k, z, \epsilon) & =\epsilon \cdot \psi(z) e^{i k_{\mu} X^{\mu}(z)} e^{\left(-\frac{Q}{2}+i y\right) \phi(z)} V_{j m}(z) e^{-\varphi_{\mathrm{gh}}(z)} \\
V_{-1}^{n}(k, z) & =\psi^{n}(z) e^{i k_{\mu} X^{\mu}(z)} e^{\left(-\frac{Q}{2}+i y\right) \phi(z)} V_{j m}(z) e^{-\varphi_{\mathrm{gh}}(z)}
\end{aligned}
$$


with $k_{\mu} k^{\mu}+\frac{Q^{2}}{4}+y^{2}+Q^{2} j(j+1)=0$.

BRST invariant vertex operators comprised of worldsheet $N=4$ massless representations are

$$
\begin{aligned}
V_{-1}(k, z, \epsilon) & =\epsilon \cdot \psi(z) e^{i k_{\mu} X^{\mu}(z)} e^{-\varphi_{\mathrm{gh}}(z)} \\
V_{-1}^{i}(k, z) & =\psi^{i}(z) e^{i k_{\mu} X^{\mu}(z)} e^{-Q \phi(z)} e^{-\varphi_{\mathrm{gh}}(z)} \\
V_{-1}^{0}(k, z) & =\psi^{0}(z) e^{i k_{\mu} X^{\mu}(z)} e^{-Q \phi(z)} e^{-\varphi_{\mathrm{gh}}(z)} \\
V_{-1}^{n}(k, z) & =\psi^{n}(z) e^{i k_{\mu} X^{\mu}(z)} e^{-\varphi_{\mathrm{gh}}(z)}
\end{aligned}
$$

with $k_{\mu} k^{\mu}=0$. It follows that states corresponding to the vertex operators in $(4.16,17)$ : $|\psi\rangle=V_{-1}(k, 0)|0\rangle$ are representations of the direct product worldsheet algebras and satisfy the physical state conditions:

$$
F_{s}|\psi\rangle=0, \text { for } s \geq \frac{1}{2} ; \quad L_{n}|\psi\rangle=0, \text { for } n \geq 1 ; \quad L_{0}|\psi\rangle=\frac{1}{2}|\psi\rangle .
$$

From $(4.17 \mathrm{~b}, \mathrm{c})$ and $(4.14)$ we see that $V_{-1}^{i}(k, 0)|0\rangle$ and $V_{-1}^{0}(k, 0)|0\rangle$ are doublets under the $S U(2)$ algebra $\overline{\mathcal{S}}_{0}^{i}$ whose affine algebra $\overline{\mathcal{S}}^{i}(z)$ are the level one $S U(2)$ generators of the $N=4, c=6$ global superconformal algebra (2.5).

From (2.8b) however, we note that the states

$$
\tilde{V}_{-1}^{i}(k, 0)|0\rangle=\psi^{i}(0) e^{i k_{\mu} X^{\mu}(0)} e^{-\varphi_{\text {gh }}(0)}|0\rangle, \quad V_{-1}^{0}(k, 0)|0\rangle
$$

are highest weight states of the $N=1$ subalgebra, and are in the adjoint and singlet representations of the $S U(2)$ algebra $T_{0}^{i}$, whose current $T^{i}(z)$ is the upper component of $\psi^{i}(z)$. Therefore $\tilde{V}_{-1}^{i}(k, 0)|0\rangle$ correspond to gauge bosons of $S U(2)$, i.e. their three-point correlation functions would have the standard non-abelian three-point coupling.

$$
i Q\left[J^{i}(z)+\frac{1}{2} \epsilon_{i k p} \psi^{k}(z) \psi^{p}(z)\right] \tilde{V}_{-1}^{j}(k, \zeta)=(z-\zeta)^{-1} Q i \epsilon_{i j k} \tilde{V}_{-1}^{k}(k, \zeta) .
$$

\section{RAMOND STATES}

The Ramond ground states of the $N=4$ superconformal algebra are expressed in terms of spin fields $S_{A}, S_{\dot{A}}$. Instead of (4.3), the $N=4$ Ramond fields satisfy

$$
\begin{aligned}
\bar{F}(z) S_{A}(\zeta) e^{\beta \phi(\zeta)} V_{j m}(\zeta)= & (z-\zeta)^{-\frac{3}{2}}\left[(\beta+Q) \frac{1}{\sqrt{2}} \gamma_{A}^{0 \dot{B}} S_{\dot{B}}(\zeta) V_{j m}(\zeta)\right. \\
& \left.-Q f_{i m m^{\prime}} \frac{1}{\sqrt{2}} \gamma_{A}^{i \dot{B}} S_{\dot{B}}(\zeta) V_{j m^{\prime}}(\zeta)\right] e^{\beta \phi(\zeta)} \\
& +O(z-\zeta)^{-\frac{1}{2}} \\
\bar{F}(z) S_{\dot{A}}(\zeta) e^{\beta \phi(\zeta)} V_{j m}(\zeta)= & (z-\zeta)^{-\frac{3}{2}}\left[\beta \frac{1}{\sqrt{2}} \gamma_{\dot{A}}^{0 B} S_{B}(\zeta) V_{j m}(\zeta)\right. \\
& \left.-Q f_{i m m^{\prime}} \frac{1}{\sqrt{2}} \gamma_{\dot{A}}^{i B} S_{B}(\zeta) V_{j m^{\prime}}(\zeta)\right] e^{\beta \phi(\zeta)} \\
+ & O(z-\zeta)^{-\frac{1}{2}}
\end{aligned}
$$


Here we choose a Weyl representation for the four-dimensional $\gamma$ matrix algebra with $\left\{\gamma^{m}, \gamma^{n}\right\}=-2 \delta^{m n}$ given by

$$
\gamma^{0}=\left(\begin{array}{cc}
0 & \left(-\sigma^{0}\right)_{\dot{B}}^{A} \\
\left(\sigma^{0}\right)_{B}^{\dot{A}} & 0
\end{array}\right) ; \quad \gamma^{j}=i\left(\begin{array}{cc}
0 & \left(\sigma^{j}\right)_{\dot{B}}^{A} \\
\left(\sigma^{j}\right)_{B}^{\dot{A}} & 0
\end{array}\right)
$$

where $\sigma^{0} \equiv\left(\begin{array}{ll}1 & 0 \\ 0 & 1\end{array}\right)$ and $\sigma^{i}$ are the Pauli matrices. The charge conjugation matrices

$$
C=\left(\begin{array}{cc}
\left(i \sigma^{2}\right)^{A B} & 0 \\
0 & \left(i \sigma^{2}\right)^{\dot{A} \dot{B}}
\end{array}\right) ; \quad C^{-1}=\left(\begin{array}{cc}
\left(-i \sigma^{2}\right)_{A B} & 0 \\
0 & \left(-i \sigma^{2}\right)_{\dot{A} \dot{B}}
\end{array}\right)
$$

raise and lower indices: $C_{A D}^{-1}\left(\gamma^{m}\right)_{\dot{B}}^{D}=\left(\gamma^{m}\right)_{A \dot{B}}$ and $C^{\dot{B} \dot{D}}\left(\gamma^{m}\right)_{\dot{D}}^{A}=\left(\gamma^{m}\right)^{A \dot{B}}$, etc. In deriving (4.20), we use the operator product expansion

$$
\begin{aligned}
\psi^{(0, i)}(z) S_{A}(\zeta) & =(z-\zeta)^{-\frac{1}{2}} \frac{1}{\sqrt{2}} \gamma_{\dot{A}}^{(0, i) \dot{B}} S_{\dot{B}}(\zeta)+\ldots \\
\psi^{(0, i)}(z) S_{\dot{A}}(\zeta) & =(z-\zeta)^{-\frac{1}{2}} \frac{1}{\sqrt{2}} \gamma_{\dot{A}}^{(0, i) B} S_{B}(\zeta)+\ldots \\
\psi^{i}(z) \psi^{j}(z) S_{A}(\zeta) & =(z-\zeta)^{-1} \frac{1}{2}\left(\gamma^{i} \gamma^{j}\right){ }_{A}^{B} S_{B}(\zeta)+\ldots, \text { etc. }
\end{aligned}
$$

and use that in this representation $\frac{1}{6} \epsilon_{i j k}\left(\gamma^{i} \gamma^{j} \gamma^{k}\right)_{A}^{\dot{B}}=-\gamma_{\dot{A}}^{0 \dot{B}}$; and $\frac{1}{6} \epsilon_{i j k}\left(\gamma^{i} \gamma^{j} \gamma^{k}\right)_{\dot{A}}^{B}=\gamma_{\dot{A}}^{0 B}$.

In the Ramond sector, the highest weight states of the $N=4$ representations are annihilated by $\bar{L}_{n}, \bar{F}_{n}, \bar{F}_{n}^{i}, \overline{\mathcal{S}}_{n}^{i}, \overline{\mathcal{S}}_{0}^{+}, \mathcal{G}_{0}^{2}, \overline{\mathcal{G}}_{0}^{1}$ for $n \geq 1$, and are labelled by

$$
\bar{L}_{0}|h, \ell\rangle=h|h, \ell\rangle \quad i \overline{\mathcal{S}}_{0}^{3}|h, \ell\rangle=\ell|h, \ell\rangle,
$$

where $\ell=\frac{1}{2}$ and $h>\frac{1}{4}$ for massive representations; and $h=\frac{1}{4}$ with $\ell=0, \frac{1}{2}$ for massless representations ${ }^{[12]}$. For massless representations, the highest weight states $|h, \ell\rangle$ are also destroyed by $\mathcal{G}_{0}^{1}$ and $\overline{\mathcal{G}}_{0}^{2}$, so that

$$
\bar{F}_{0}|h, \ell\rangle=0 ; \quad \bar{F}_{0}^{i}|h, \ell\rangle=0
$$

From (4.22), we find the global level one $S U(2)$ current acts on the spin field states as

$$
i \overline{\mathcal{S}}_{0}^{3} S_{1}(0)|0\rangle=-\frac{1}{2} S_{1}(0)|0\rangle, \quad i \overline{\mathcal{S}}_{0}^{3} S_{2}(0)|0\rangle=\frac{1}{2} S_{2}(0)|0\rangle ; \quad i \overline{\mathcal{S}}_{0}^{3} S_{\dot{A}}(0)|0\rangle=0
$$

and from (2.5) and (4.20,22), we have that in the Ramond sector, the highest weight $N=4$ massive state with $h>\frac{1}{4}, \ell=\frac{1}{2}$ is $S_{2}(0) e^{\left.\left(-\frac{Q}{2}+i y\right) \phi(0)\right)} V_{00}(0)|0\rangle$.

The highest weight $N=4$ massless Ramond states with $h=\frac{1}{4}$ are, for $\ell=\frac{1}{2}$, $S_{A}(0) e^{-Q \phi(0))}|0\rangle$; and $S_{\dot{A}}(0)|0\rangle$ for $\ell=0$.

It is convenient to bosonize the free fermions in terms of two bosons, $H^{ \pm}(z)$ as

$$
\overline{\mathcal{S}}^{3}=\frac{1}{\sqrt{2}} \partial H^{+}, \quad \overline{\mathcal{S}}^{ \pm}=e^{ \pm i \sqrt{2} H^{+}} ; \quad \tilde{\mathcal{S}}^{3}=\frac{1}{\sqrt{2}} \partial H^{-}, \quad \tilde{\mathcal{S}}^{ \pm}=e^{ \pm i \sqrt{2} H^{-}}
$$


where

$$
\overline{\mathcal{S}}^{i}=\frac{1}{2}\left(\psi^{0} \psi^{i}+\frac{1}{2} \epsilon_{i j \ell} \psi^{j} \psi^{\ell}\right) ; \quad \tilde{\mathcal{S}}^{i}=\frac{1}{2}\left(-\psi^{0} \psi^{i}+\frac{1}{2} \epsilon_{i j \ell} \psi^{j} \psi^{\ell}\right)
$$

are two commuting level one $S U(2)$ currents. Since

$$
i \overline{\mathcal{S}}^{3}(z) e^{ \pm \frac{i}{\sqrt{2}} H^{+}(\zeta)}= \pm \frac{1}{2}(z-\zeta)^{-1} e^{ \pm \frac{i}{\sqrt{2}} H^{+}(\zeta)} ; \quad i \overline{\mathcal{S}}^{3}(z) e^{ \pm \frac{i}{\sqrt{2}} H^{-}(\zeta)}=0
$$

we identify

$$
S_{A}(z)=e^{\mp \frac{i}{\sqrt{2}} H^{+}(z)} \quad S_{\dot{A}}(z)=e^{\mp \frac{i}{\sqrt{2}} H^{-}(z)} .
$$

In general, modular invariance requires certain projections of the spin fields which restrict the physical spectrum. In the direct product of the three conformal field theories: ( spacetime $\mathrm{cft} c=6) \otimes($ fivebrane $\mathrm{cft} c=6) \otimes\left(T^{2} \mathrm{cft} c=3\right)$, we consider the BRST invariant weight one primary conformal fields for Ramond ground states in the superconformal ghost number $q=-\frac{1}{2}$ picture comprised of $N=4$ massless highest weight representations given by

$$
\begin{aligned}
& V_{-\frac{1}{2}}^{(1)}(k, z)=u^{1 \alpha}(k) S_{\alpha}(z) e^{i k_{\mu} X^{\mu}(z)} S_{A}(z) e^{-Q \phi(z)} S_{n}(z) e^{-\frac{1}{2} \varphi_{\mathrm{gh}}(z)} \\
& V_{-\frac{1}{2}}^{(2)}(k, z)=u^{2 \dot{\alpha}}(k) S_{\dot{\alpha}}(z) e^{i k_{\mu} X^{\mu}(z)} S_{A}(z) e^{-Q \phi(z)} S_{\dot{n}}(z) e^{-\frac{1}{2} \varphi_{\mathrm{gh}}(z)}
\end{aligned}
$$

where $k_{\mu} k^{\mu}=0 ; S_{\alpha}, S_{\dot{\alpha}}$ are the spin fields of 4-dimensional spacetime, and $S_{n}, S_{\dot{n}}$, $n=1, \dot{n}=\dot{1}$ are the weight $\frac{1}{8}$ spin fields associated with the fermionic component $\psi^{\ell}$ of the supercoordinates on $T^{(2)}$. In (4.30), the two linearly independent solutions to the massless Dirac equation $k \cdot \gamma u(k)=0$ are given by solutions of the Weyl equations

$$
k_{\mu} \sigma_{\beta}^{\mu \dot{\alpha}} u^{1 \beta}=0 \quad k_{\mu} \bar{\sigma}_{\dot{\beta}}^{\mu \alpha} u^{2 \dot{\beta}}=0
$$

and $\bar{\sigma}^{0}=-\sigma^{0}, \bar{\sigma}^{i}=\sigma^{i}$.

From $(4.25,4.30)$, we see that the Ramond states $V_{-\frac{1}{2}}^{(1,2)}(k, 0)|0\rangle$ each correspond to a doublet under the level one $S U(2)$ algebra $\overline{\mathcal{S}}_{0}^{i}$. Since

$$
\begin{aligned}
& i T^{i}(z) S_{A}(\zeta)=(z-\zeta)^{-1} Q \frac{i}{4} \epsilon_{i j k}\left(\gamma^{j} \gamma^{k}\right){ }_{A}^{B} S_{B}(\zeta) \\
& i T^{i}(z) S_{\dot{A}}(\zeta)=(z-\zeta)^{-1} Q \frac{i}{4} \epsilon_{i j k}\left(\gamma^{j} \gamma^{k}\right)_{\dot{A}}^{\dot{B}} S_{\dot{B}}(\zeta)
\end{aligned}
$$

we have

$$
\begin{array}{cl}
i T_{0}^{3} S_{1}(0)|-Q\rangle=-Q \frac{1}{2} S_{1}(0)|-Q\rangle, & i T_{0}^{3} S_{2}(0)|-Q\rangle=Q \frac{1}{2} S_{2}(0)|-Q\rangle \\
i T_{0}^{3} S_{\dot{1}}(0)|0\rangle=-Q \frac{1}{2} S_{\dot{1}}(0)|0\rangle, & i T_{0}^{3} S_{\dot{2}}(0)|0\rangle=Q \frac{1}{2} S_{\dot{2}}(0)|0\rangle,
\end{array}
$$

so that $V_{-\frac{1}{2}}^{(1,2)}(k, 0)|0\rangle$ each also correspond to a doublet under the $S U(2)$ algebra $T_{0}^{i}$. 
Therefore these fermion states, which are highest weight states of massless representations of the $N=4$ algebra and consequently of the $N=1$ subalgebra, carry charge under an $S U(2)$ gauge algebra. In the next section, we discuss the implications of this for representations of the $N=1$ super Kac-Moody algebra.

\section{Fivebrane representation of $N=1$ SVA and SKMA}

The construction of the fivebrane $c=6, N=1$ superVirasoro algebra (SVA) (which is a subalgebra of the $N=4$ system) given by

$$
\begin{aligned}
& L(z)=-\frac{1}{2} J^{0} J^{0}-\frac{1}{k+2} J^{i} J^{i}-\frac{1}{2} \partial \psi^{A} \psi^{A}+\delta \frac{1}{4 z^{2}}+\frac{1}{2} Q \partial J^{0} \\
& F(z)=\psi^{0} J^{0}-Q \psi^{i} J^{i}-Q \frac{1}{6} \epsilon_{i j k} \psi^{i} \psi^{j} \psi^{k}-Q \partial \psi^{0}
\end{aligned}
$$

for $\delta=0,1$ for NS or R sector respectively, together with the weight one-half superfield whose components are

$$
\psi^{i}(z), \quad T^{i}(z)=Q\left[J^{i}+\frac{1}{2} \epsilon_{i j k} \psi^{j} \psi^{k}\right]
$$

form a representation of the super $S U(2)$ Kac-Moody algebra (SKMA) mixing with the $N=1$ SVA:

$$
\begin{aligned}
F(z) \psi^{i}(\zeta) & =(z-\zeta)^{-1} T^{i}(\zeta), \quad F(z) T^{i}(\zeta)=(z-\zeta)^{-2} \psi^{i}(\zeta)+(z-\zeta)^{-1} \partial \psi^{i}(\zeta) \\
T^{i}(z) T^{j}(\zeta) & =-\frac{\delta_{i j}}{(z-\zeta)^{2}}+\frac{Q \epsilon_{i j k} T^{k}(\zeta)}{(z-\zeta)} \\
T^{i}(z) \psi^{j}(\zeta) & =\frac{Q \epsilon_{i j k} \psi^{k}(\zeta)}{(z-\zeta)}, \quad \psi^{i}(z) \psi^{j}(\zeta)=-\frac{\delta_{i j}}{(z-\zeta)}
\end{aligned}
$$

where $J^{i}$ is the level $k \mathrm{SU}(2) \mathrm{KMA}$ and $Q=-\sqrt{\frac{2}{(k+2)}}$. We note that superfield associated with $\psi^{0}(z)$ has lower and upper components $\psi^{0}(z) e^{-Q \phi(z)}, \quad T^{0}(z)=-\frac{1}{Q} \partial_{z} e^{-Q \phi(z)}$ so that unless $Q=0$ the current $T^{0}(z)$ is not the generator of a $U(1)$ Heisenberg algebra.

For simplicity, we can consider $k=0$, in which case $Q=-1$ and the currents $J^{i}$ decouple from the system, so that (5.1-5.5) become with $c=6$ :

$$
\begin{aligned}
& L(z)=-\frac{1}{2} J^{0} J^{0}-\frac{1}{2} \partial \psi^{A} \psi^{A}+\delta \frac{1}{4 z^{2}}-\frac{1}{2} \partial J^{0} \\
& F(z)=\psi^{0} J^{0}+\frac{1}{6} \epsilon_{i j k} \psi^{i} \psi^{j} \psi^{k}+\partial \psi^{0} \\
& \psi^{i}(z), \quad T^{i}(z)=-\frac{1}{2} \epsilon_{i j k} \psi^{j} \psi^{k} \\
& F(z) \psi^{i}(\zeta)=(z-\zeta)^{-1} T^{i}(\zeta), \quad F(z) T^{i}(\zeta)=(z-\zeta)^{-2} \psi^{i}(\zeta)+(z-\zeta)^{-1} \partial \psi^{i}(\zeta) \\
& T^{i}(z) T^{j}(\zeta)=-\frac{\delta_{i j}}{(z-\zeta)^{2}}-\frac{\epsilon_{i j k} T^{k}(\zeta)}{(z-\zeta)} \\
& T^{i}(z) \psi^{j}(\zeta)=-\frac{\epsilon_{i j k} \psi^{k}(\zeta)}{(z-\zeta)}, \quad \psi^{i}(z) \psi^{j}(\zeta)=-\frac{\delta_{i j}}{(z-\zeta)} .
\end{aligned}
$$


For the system (5.6), we find that the Ramond states $S_{A}(0) e^{-Q \phi(0)}|0\rangle$ and $S_{\dot{A}}(0)|0\rangle$ satisfy

$$
F_{0} S_{A}(0)|-Q\rangle=0, \quad F_{0} S_{\dot{A}}(0)|0\rangle=0
$$

but that they are carry non-trivial $S U(2)$ charge:

$$
\begin{aligned}
& -i\left\{F_{0}, \psi_{0}^{3}\right\} S_{1}(0)|-Q\rangle=-i T_{0}^{3} S_{1}(0)|-Q\rangle=-\frac{1}{2} S_{1}(0)|-Q\rangle \\
& -i\left\{F_{0}, \psi_{0}^{3}\right\} S_{2}(0)|-Q\rangle=-i T_{0}^{3} S_{2}(0)|-Q\rangle=\frac{1}{2} S_{2}(0)|-Q\rangle \\
& -i\left\{F_{0}, \psi_{0}^{3}\right\} S_{\dot{1}}(0)|0\rangle=-i T_{0}^{3} S_{\dot{1}}(0)|0\rangle=-\frac{1}{2} S_{\dot{1}}(0)|0\rangle \\
& -i\left\{F_{0}, \psi_{0}^{3}\right\} S_{\dot{2}}(0)|0\rangle=-i T_{0}^{3} S_{\dot{2}}(0)|0\rangle=+\frac{1}{2} S_{\dot{2}}(0)|0\rangle .
\end{aligned}
$$

Since these Ramond states are massless representations of the $N=4$ algebra, they are also representations of the $N=1$ subalgebra given in (5.6). In this way, in a closed string theory, a non-abelian $S U(2)$ gauge symmetry can occur on either side which supports massless charged fermions. This evades the argument of the DKV theorem ${ }^{[18]}$, which had prevented massless (R,NS) fermions in a string model with a left-moving super Kac-Moody algebra. Furthermore these fermions carry left-moving $S U(2)$ charge. Given (5.16) and (2.19), these states have positive norm.

The mechanism which allows for massless fermions charged under an $S U(2)$ gauge symmetry occurring in the Ramond sector is the choice of inner product (2.19) and it works as follows: From (5.7),(5.8a) we have

$$
i F_{0} \psi_{0}^{3} S_{1}(0)|-Q\rangle=i T_{0}^{3} S_{1}(0)|-Q\rangle=\frac{1}{2} S_{1}(0)|-Q\rangle
$$

since $F_{0} S_{1}(0)|-Q\rangle=0$ from (5.7). As in (5.16), taking the norm of the righthand side of (5.9), we have

$$
\frac{1}{2} \lim _{z \rightarrow \infty} z^{\frac{1}{2}}\left\langle 0\left|S^{1}(z) S_{1}(0)\right|-Q\right\rangle=\frac{1}{2} .
$$

It follows that this action on the lefthand side of (5.9) is also non-zero:

$$
\lim _{z \rightarrow \infty} z^{\frac{1}{2}}\left\langle 0\left|S^{1}(z) F_{0} i \psi_{0}^{3} S_{1}(0)\right|-Q\right\rangle=\lim _{z \rightarrow \infty} z^{\frac{1}{2}}\left\langle 0\left|S^{1}(z) F_{0}\left(-\frac{1}{\sqrt{2}}\right) S_{\mathrm{i}}(0)\right|-Q\right\rangle=\frac{1}{2}
$$

SO

$$
\lim _{z \rightarrow \infty} z^{\frac{1}{2}}\langle 0| S^{1}(z) F_{0}=\left(-\frac{1}{\sqrt{2}}\right) \lim _{z \rightarrow \infty} z^{\frac{1}{2}}\langle 0| S^{\dot{1}}(z) \neq 0
$$

which is the same statement as

$$
\left[\lim _{z \rightarrow \infty} z^{\frac{1}{2}}\langle 0| S^{1}(z) F_{0}\right]^{\dagger}=\left(-\frac{1}{\sqrt{2}}\right) S_{\mathrm{i}}(0)|-Q\rangle \neq 0 .
$$

Therefore, although

$$
F_{0} S_{1}(0)|-Q\rangle=0,
$$

we have that $F_{0}$ acting on the state adjoint to $S_{1}(0)|-Q\rangle$ is not zero, as shown in (5.12),(5.13). This is allowed, since although the hermiticity property of the inner product requires

$$
\begin{aligned}
& 0=\left(S_{1}(0)|-Q\rangle, F_{0} S_{1}(0)|-Q\rangle\right)=\left(\left[\lim _{z \rightarrow \infty} z^{\frac{1}{2}}\langle 0| S^{1}(z) F_{0}\right]^{\dagger}, S_{1}(0)|-Q\rangle\right) \\
& =\lim _{z \rightarrow \infty} z^{\frac{1}{2}}\left\langle 0\left|S^{1}(z) F_{0} S_{1}(0)\right|-Q\right\rangle=-\frac{1}{\sqrt{2}} \lim _{z \rightarrow \infty} z^{\frac{1}{2}}\left\langle 0\left|S^{\mathrm{i}}(z) S_{1}(0)\right|-Q\right\rangle=-\frac{\delta_{1}^{\mathrm{i}}}{\sqrt{2}}=0,
\end{aligned}
$$


the righthand side of (5.15a) vanishes because the states in the inner product are orthogonal, not because $\left[\lim _{z \rightarrow \infty} z^{\frac{1}{2}}\langle 0| S^{1}(z) F_{0}\right]^{\dagger}$ is zero.

The norm of the spin field states $|\psi\rangle \equiv S_{A}(0)|0\rangle$ without the Liouville contribution is given by ${ }^{[19]}$

$$
\begin{aligned}
\langle\psi \mid \psi\rangle & =\lim _{\substack{z \rightarrow \infty \\
\zeta \rightarrow 0}} z^{2 h}\langle 0|V(\bar{\psi}, z) V(\psi, \zeta)| 0\rangle=\lim _{\substack{z \rightarrow \infty \\
\zeta \rightarrow 0}} z^{\frac{1}{2}}\left\langle 0\left|S^{A}(z) S_{A}(\zeta)\right| 0\right\rangle \\
& =\lim _{\substack{z \rightarrow \infty \\
\zeta \rightarrow 0}} z^{\frac{1}{2}}(z-\zeta)^{-\frac{1}{2}} C^{A B} C_{B A}^{-1}=\delta_{A}^{A}=1,
\end{aligned}
$$

where the vertex for the state $\bar{\psi}$ conjugate to $\psi$ is given by $V(\bar{\psi}, z) \equiv z^{-2 h} V\left(\psi, \frac{1}{z^{*}}\right)^{\dagger}$. Since $S_{A n}^{\dagger}=S_{-n}^{A}$, the state conjugate to $S_{A}(0)|0\rangle$ is $S^{A}(0)|0\rangle$. We have ${ }^{[19]}$

$$
\begin{aligned}
\psi=\lim _{z \rightarrow 0} V(\psi, z)|0\rangle, & \bar{\psi}=\lim _{z \rightarrow 0} V(\bar{\psi}, z)|0\rangle \\
\langle\bar{\psi}|=\lim _{z \rightarrow \infty} z^{2 h}\langle 0| V(\psi, z), & \langle\psi|=\lim _{z \rightarrow \infty} z^{2 h}\langle 0| V(\bar{\psi}, z) .
\end{aligned}
$$

For the discrete Liouville states $|q\rangle=e^{q \phi(0)}|0\rangle$, the non-vanishing inner product is $\langle-q-Q \mid q\rangle=1$. As in sect. 2, we define the adjoint of $|q\rangle=e^{q \phi(0)}|0\rangle$ to be $\langle-q-Q|$, so that the state $|q\rangle$ has non-zero norm: $\||q\rangle \|^{2}=\langle-q-Q \mid q\rangle=1$. Clearly all propagating states, i.e. those which have continuous momenta such as (4.30) with $k_{\mu} k^{\mu}=0$ but $k^{\mu} \neq 0$, must have positive norm. This completes our discussion of the existence of charged massless Ramond states. ( In (5.6)-(5.15) we choose $Q=-1$. The discussion is straightforwardly generalized for any real $Q=-\sqrt{\frac{2}{k+2}}$. The limit $Q \rightarrow 0$ collapses to zero charge fermions.)

The set of consistent GSO projections for states such as (4.30) in a string theory with space-time perturbative unitarity is being investigated. This may include projecting away massive states such as (4.16) which depend on a continous parameter $y .{ }^{[20]}$ We note here that another set of BRST invariant Ramond operators is given by

$$
\begin{aligned}
V_{-\frac{1}{2}}^{\prime(1)}(k, z)= & {\left[u^{1 \alpha}(k) S_{\alpha}(z) S_{A}(z) e^{-Q \phi(z)}-Q v^{1 \dot{\alpha}}(k) S_{\dot{\alpha}}(z) \gamma_{A}^{0 \dot{A}} S_{\dot{A}}(z)\right] } \\
& \cdot S_{n}(z) e^{i k_{\mu} X^{\mu}(z)} e^{-\frac{1}{2} \varphi_{\mathrm{gh}}(z)}
\end{aligned}
$$

with a similar expression for $V_{-\frac{1}{2}}^{(2)}(k, z)$. These vertex operators, associated with different projections from (4.30), give rise to non-abelian tree amplitudes of Ramond-Ramond states. ${ }^{[21]}$ Here we defined two additional spinors $v^{\ell}(k)$ by $k \cdot \gamma v^{\ell} \sim u^{\ell}$, i.e.

$$
\frac{1}{\sqrt{2}} k_{\mu} \bar{\sigma}_{\dot{\beta}}^{\mu \alpha} v^{1 \dot{\beta}}=u^{1 \alpha} \quad \frac{1}{\sqrt{2}} k_{\mu} \sigma_{\beta}^{\mu \dot{\alpha}} v^{2 \beta}=-u^{2 \dot{\alpha}}
$$

\section{Conclusions}

The specific fivebrane CFT permits us to compute the spectrum of string excitations around the corresponding background solution. In this sense, the choice of CFT provides non-perturbative information. 
In formulations of string theory, the question arises as to whether 1) all the necessary information can be seen as the spectrum of some CFT, or instead 2) does a framework broader than CFT need to be employed to realize the natural string ground state. In this paper, we investigate only the first point of view, but we point to effects which occur in CFTs, albeit unconventional ones with background charge, which lead to novel gauge properties of the spectrum. This is in the spirit that in order to solve any non-linear system, ultimately a perturbation theory about some new point will do the job. In this paper, that new point is the Type II superstring with an internal background charge CFT.

The fact that in physics the massless spectrum, i.e. the low mass spectrum carries representations of the gauge group, and that the gauge generators are the zero modes of weight one conformal fields, suggests that at least the massless states should be described by some CFT.

In this paper, we were led to consider a conformal field theory with background charge in order to have charged Ramond states. The fivebrane conformal field theory provides a specific example of such a conformal field theory, and its use was motivated by the fact that the fivebrane, an NS-NS brane, carries charge under the NS gauge bosons. Furthermore, a connection between gauged supergravity theories ${ }^{[22-26]}$ which necessarily have tree level cosmological constants, and string theories ${ }^{[21,11]}$ serves to increase interest in pursuing non-critical string theories ${ }^{[10,11]}$. The latter provide a link between string theory and field theories with tree level cosmological constants.

The presence of gauge symmetry in string theory is closely tied to affine Kac-Moody $\operatorname{algebras}^{[27-29]}$. We showed in sect.5 how a choice of inner product for the discrete Liouville states in this CFT leads to a generalization of representations of the $N=1$ super $S U(2)$ Kac-Moody algebra. In principle, this now allows an $S U(2)$ non-abelian factor in the gauge group on one side, with the original non-abelian factors (like $S U(3) \otimes U(1)$ ) coming from the other side, and provides symmetry enhancement within the economical Type II superstring.

The author acknowledges valuable discussions with A. Belopolsky, R. Blumenhagen, M. Flohr, P. di Francesco, and P. Goddard, and is grateful for the hospitality of St.John's College, Cambridge where part of this work was done. 


\section{Appendix}

$N=4, c=6$ SUPERCONFORMAL ALGEBRA

The currents of (2.5) close on the $N=4$ superconformal algebra with $c=6$ which is given by

$$
\begin{aligned}
& L(z) L(\zeta)=\frac{\frac{c}{2}}{(z-\zeta)^{4}}+\frac{2 L(\zeta)}{(z-\zeta)^{2}}+\frac{\partial L(\zeta)}{(z-\zeta)} \\
& L(z) F(\zeta)=\frac{\frac{3}{2} F(\zeta)}{(z-\zeta)^{2}}+\frac{\partial F(\zeta)}{(z-\zeta)} \\
& L(z) F^{i}(\zeta)=\frac{\frac{3}{2} F^{i}(\zeta)}{(z-\zeta)^{2}}+\frac{\partial F^{i}(\zeta)}{(z-\zeta)} \\
& L(z) S^{i}(\zeta)=\frac{S^{i}(\zeta)}{(z-\zeta)^{2}}+\frac{\partial S^{i}(\zeta)}{(z-\zeta)} \\
& F(z) F(\zeta)=\frac{\frac{2 c}{3}}{(z-\zeta)^{3}}+\frac{2 L(\zeta)}{(z-\zeta)} \\
& F(z) F^{i}(\zeta)=-\frac{4 S^{i}(\zeta)}{(z-\zeta)^{2}}-\frac{2 \partial S^{i}(\zeta)}{(z-\zeta)} \\
& F^{i}(z) F^{j}(\zeta)=\frac{\delta^{i j} \frac{2 c}{3}}{(z-\zeta)^{3}}-\frac{4 \epsilon_{i j \ell} S^{\ell}(\zeta)}{(z-\zeta)^{2}}-\frac{2 \epsilon_{i j \ell} \partial S^{\ell}(\zeta)}{(z-\zeta)}+\frac{2 \delta^{i j} L(\zeta)}{(z-\zeta)} \\
& S^{i}(z) S^{j}(\zeta)=-\frac{n \delta^{i j}}{2(z-\zeta)^{2}}+\frac{\epsilon_{i j \ell} S^{\ell}(\zeta)}{(z-\zeta)} \\
& S^{i}(z) F(\zeta)=\frac{F^{i}(\zeta)}{2(z-\zeta)} \\
& S^{i}(z) F^{j}(\zeta)=\frac{1}{(z-\zeta)}\left[-\delta^{i j} F(\zeta)+\epsilon_{i j \ell} F^{\ell}(\zeta)\right] .
\end{aligned}
$$

The central charge $c$ and the level $n$ of the $S U(2)_{n}$ currents $S^{i}$ are related by $c=6 n$. The condition $c=6$ sets $S^{i}$ at level one.

We define complex supercurrents as

$$
\begin{aligned}
& \mathcal{G}^{1} \equiv \frac{F-i F^{3}}{\sqrt{2}}, \quad \mathcal{G}^{2} \equiv \frac{F^{2}-i F^{1}}{\sqrt{2}} \\
& \overline{\mathcal{G}}^{1} \equiv \frac{F+i F^{3}}{\sqrt{2}}, \quad \overline{\mathcal{G}}^{2} \equiv \frac{F^{2}+i F^{1}}{\sqrt{2}}
\end{aligned}
$$

and note that $\mathcal{G}^{1}, \mathcal{G}^{2}$ transform as a doublet under $S^{i}$ etc. 


\section{References}

1. A. Strominger, Heterotic solitons, Nucl. Phys. B343 (1990) 167.

2. C. Callan, J. Harvey, and A. Strominger, Worldsheet approach to heterotic instantons and solitons, Nucl. Phys. B359 (1991) 611; and Worldbrane actions for string solitons, Nucl. Phys. B 367 (1991) 60.

3. M.Duff, R. Khuri, and J.X. Lu, String solitions, Phys. Rep. 259 (1995) 213-326, hep-th/9412184.

4. C. Callan, Instantons and solitons in heterotic string theory, Swieca Summer School, June 1991; hep-th/9109052. C. Callan, J. Harvey, and A. Strominger, Supersymmetric string solitions, 1991 Trieste Spring School on String Theory and Quantum Gravity, hep-th/9111030.

5. A. Sevrin, W. Troost, and A. van Proyen, Superconformal algebras in two dimensions with $N=4$, Phys. Lett. B208 (1988) 447.

6. C. Hull and P. Townsend, Nucl. Phys. B438 (1995) 109, hep-th/9410167; Nucl. Phys. B451 (1995) 525, hep-th/9505073.

7. E. Witten, String theory in various dimensions, Nucl. Phys. B443 (1995) 85, hepth/9503124.

8. J. Polchinski, Dirichlet-Branes and Ramond-Ramond Charges, Phys. Rev. Lett. 75 (1995) 4727, hep-th/9510017.

9. P. Townsend, D-branes from M-branes, Phys. Lett. B373 (1996) 68, hep-th/9512062.

10. N. Seiberg and D. Kutasov, Non-critical superstrings, Phys. Lett. B251 (1990) 67.

11. I. Antoniadis, S. Ferrara, C. Kounnas, Exact supersymmetric string solutions in curved gravitational backgrounds, Nucl. Phys.B421 (1994) 343, hep-th/9402073.

12. T. Eguchi and A. Taormina, Unitary representations of the $N=4$ superconformal algebra, Phys. Lett. B196 (1986) 75; Character formulas for the $N=4$ superconformal algebra, Phys. Lett. B200 (1988) 315.

13. G. Mostow and J. Sampson, Linear Algebra, New York: McGraw-Hill, 1969.

14. T. Banks, J. Dixon, D. Friedan, and E. Martinec, Nucl. Phys. B299 (1988) 613.

15. T. Banks, J. Dixon, Nucl. Phys. B307 (1988) 93.

16. D. Friedan, E. Martinec, and S. Shenker, Nucl. Phys. B271 (1986) 93.

17. I. Klebanov and A. Polyakov, Mod. Phys. Lett A6(1991)635 hep-th/9109032; I. Klebanov and A Pasquinucci, hep-th/9210105.

18. L. Dixon, V. Kaplunovsky and C. Vafa, Nucl. Phys. B294 (1987) 43.

19. R. Bluhm, L. Dolan and P. Goddard, Conformal field theory of twisted vertex opera- 
tors, Nucl. Phys. B338 (1990) 529.

20. L. Dolan, work in progress.

21. L. Dolan and S. Horvath, Nucl. Phys. B448 (1995) 220.

22. E. Cremmer and B. Julia, "The SO(8) Supergravity", Nucl. Phys.B159 (1979) 141.

23. B. de Wit and H. Nicolai, " $N=8$ supergravity with local $S O(8) \otimes S U(8)$ invariance", Phys. Lett. B108 (1982) 285; "N=8 Supergravity", Nucl. Phys.B208 (1982) 323.

24. M.Duff, B. Nilsson, and C. Pope, "Kaluza-Klein Supergravity", Phys. Rep. 130 (1986) 1.

25. L. Romans, Phys. Lett. B169 (1986) 374.

26. I. Antoniadis, C. Bachas, and A. Sagnotti, Phys. Lett. B235 (1990) 255.

27. R. Bluhm, L. Dolan and P. Goddard, Nucl. Phys. B289 364 (1987); Nucl. Phys. B309 330 (1988).

28. M.B. Green, J. Schwarz and E. Witten, Superstring theory (Cambridge University Press, 1987).

29. L. Dolan, "The Beacon of Kac-Moody Symmetry for Physics", Notices of the American Mathematical Society, 42 No. 12, 1489-1495, December 1995. 